\title{
Erratum
}

\section{Erratum to: Multidimensional Potential Burgers Turbulence}

\author{
Alexandre Boritchev \\ CNRS UMR 5208, Institut Camille Jordan, University of Lyon, University Claude Bernard Lyon 1, 43 Blvd. \\ du 11 novembre 1918, 69622 Villeurbanne Cedex, France. E-mail: alexandre.boritchev@gmail.com
}

Received: 11 January 2016/ Accepted: 4 March 2016

Published online: 15 April 2016 - (C) Springer-Verlag Berlin Heidelberg 2016

\section{Erratum to: Commun. Math. Phys. 342, 441-489 (2016) DOI 10.1007/s00220-015-2521-7}

In the original article we overlooked that the space $L_{\infty} / \mathbb{R}$ on which we consider the dual-Lipschitz metric is not separable: thus we are not in the standard setting in which stationary measures for stochastic PDEs are studied (cf. [1]). Moreover, since $C^{\infty}$ is not dense in $L_{\infty}$, the semigroup $S_{t}^{\omega}$ is not well-defined on the space of functions in $L_{\infty}$ defined modulo an additive constant $L_{\infty} / \mathbb{R}$. Thus Lemma 8.4. does not hold.

However, we can consider the separable space of continuous functions modulo an additive constant $C^{0} / \mathbb{R}$. In this setting, the semigroups $S_{t}^{\omega}$ and $S_{t}^{*}$ are well-defined. The proof of the following result is almost word-for-word the same as the proof of the corresponding 1d $L_{1}$-nonexpansion result for the stochastic Burgers equation in [2].

Lemma. There exist positive constants $C^{\prime}, \delta$ such that for $\psi_{1}^{0}, \psi_{2}^{0} \in C^{0}$ we have

$$
\mathbf{E}\left|S_{t}^{\omega} \psi_{1}^{0}-S_{t}^{\omega} \psi_{2}^{0}\right|_{C^{0} / \mathbb{R}} \leq C^{\prime} t^{-\delta}, \quad t \geq 1
$$

In particular, these constants do not depend on $\psi_{1}^{0}, \psi_{2}^{0}$.

For all $\omega$ the solution of the stochastic Burgers equation is $C^{\infty}$-smooth in space for $t>0$ : this is proved in Appendix 1 . This allows us to define the semigroups $\tilde{S}_{t}^{\omega}$ and $\tilde{S}_{t}^{*}$, acting respectively on $L_{1}$ and on the space of probability measures on $L_{1}$. Indeed, first we consider two solutions $\psi_{1}, \psi_{2}$ to the stochastic Hamilton-Jacobi equation with the same noise and different smooth initial conditions, as well as the corresponding solutions 
$\mathbf{u}_{1}, \mathbf{u}_{2}$ to the stochastic Burgers equation. By the Gagliardo-Nirenberg inequality we get

$$
\begin{aligned}
\left|\mathbf{u}_{1}-\mathbf{u}_{2}\right|_{1} & \leq C\left|\psi_{1}-\psi_{2}-\int_{\mathbf{T}^{d}}\left(\psi_{1}-\psi_{2}\right)\right|_{1}\left|\nabla\left(\psi_{1}-\psi_{2}\right)\right|_{1,1} \\
& \leq C\left|\psi_{1}-\psi_{2}-\int_{\mathbf{T}^{d}}\left(\psi_{1}-\psi_{2}\right)\right|_{\infty}\left|\mathbf{u}_{1}-\mathbf{u}_{2}\right|_{1,1} .
\end{aligned}
$$

Thus, using Theorem 6.2. and the lemma stated above we obtain the existence of a $u_{1}^{0}, u_{2}^{0}$-independent constant $C^{\prime}$ such that:

$$
\mathbf{E}\left|\tilde{S}_{t}^{\omega} \psi_{1}^{0}-\tilde{S}_{t}^{\omega} \psi_{2}^{0}\right|_{L_{1}} \leq C^{\prime} t^{-\delta / 2}, \quad t \geq 1,
$$

with the same $\delta$ as above. These inequalities allows us first to prove by density of $C^{\infty}$ in $L_{1}$ that $\tilde{S}_{t}^{\omega}$ and $\tilde{S}_{t}^{*}$ are well-defined respectively on $L_{1}$ and on the space of probability measures on $L_{1}$, and then to obtain Theorem 8.5.

As a conclusion, while Lemma 8.4. does not hold, all other results remain valid.

\section{References}

1. Kuksin, S., Shirikyan, A.: Mathematics of two-dimensional turbulence. In: Cambridge Tracts in Mathematics, vol. 194. Cambridge University Press, Cambridge (2012)

2. Boritchev, A.: Sharp estimates for turbulence in white-forced generalised Burgers equation. GAFA 23(6), 1730-1771 (2013)

Communicated by H. Spohn 